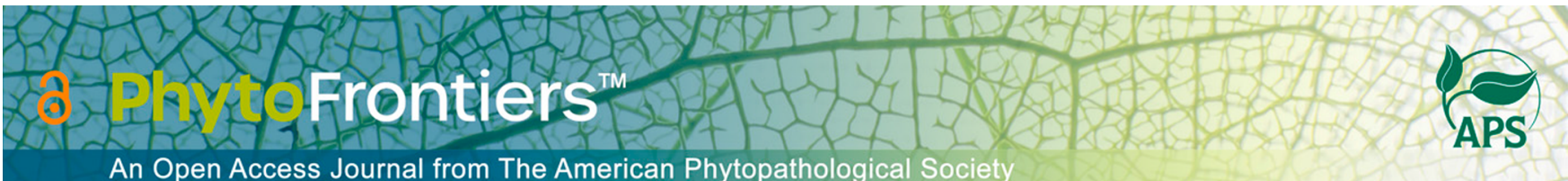

\title{
Research
}

\section{Genomic Sequence Data of Bacterial Isolates from Pistachio Trees and Other Woody Plants in California Are Inconsistent with the Role of Rhodococcus as the Causative Agent of Pistachio Bushy Top Syndrome}

\author{
Brendan K. Riely ${ }^{1,+}$ (D) | Mohamed T. Nouri² ${ }^{2}$ | Kashif Riaz ${ }^{1}$ | Muhammad Rizwan Tufail ${ }^{1}$ | \\ Yunpeng Gai ${ }^{1}$ | Noelia Carrasquilla ${ }^{1}$ | Amna Fayyaz ${ }^{1}$ | Peter L. Chang ${ }^{1}$ | Johan H. J. Leveau ${ }^{1}$ (i) | \\ Douglas R. Cook ${ }^{1}$ | Florent P. Trouillas ${ }^{1,3,+}$ (1) |
}

\footnotetext{
1 Department of Plant Pathology, University of California, Davis, CA 95616

2 University of California Cooperative Extension San Joaquin County, Stockton, CA 95206

${ }^{3}$ Kearney Agricultural Research and Extension Center, Parlier, CA 93648
}

\section{$\dagger$ Corresponding authors: F. P. Trouillas; flotrouillas@ucanr.edu, and B. K. Riely; bkriely@ucdavis.edu}

Accepted for publication 7 October 2021

Data availability: The genomes described in this manuscript have been submitted to $\mathrm{NCBI}$ and the BIOPROJECT, and BIOSAMPLE numbers are provided in Supplementary Table S1.

\section{Funding}

This work was funded by California Pistachio Research Board awards A180514 and A19-3974

e-Xtra: Two supplementary figures and four supplementary tables are available online.

The author(s) declare no conflict of interest.

\begin{abstract}
Pistachio bushy top syndrome (PBTS) is a serious problem for pistachio growers in the western United States, but the cause of this disorder remains controversial. Recently, it was proposed that the Rhodococcus species $R$. fascians and $R$. corynebacterioides caused PBTS outbreaks in 2011 and 2015. To investigate the association of Rhodococcus spp. with PBTS in California's pistachio-growing region, Rhodococcus-like isolates were collected from diverse hosts and environments, including pistachio nurseries and orchards. Whole genome sequence analysis of 231 isolates revealed their evolutionary relationships and identified six Rhodococcus species. Combined with data on geography and host of origin, the data reveal that $R$ hodococcus generally, and $R$. fascians specifically, is ubiquitous in nature, frequently occurring in both symptomatic and asymptomatic pistachio trees and on other woody and native species. Core gene and single nucleotide polymorphism-based phylogenies and pangenome analyses differentiate $R$. fascians into distinct genotypes. Although we found examples of common genotypes shared between nurseries and orchards, the observed patterns are most consistent with an environmental source of strains and do not support a scenario in which individual nurseries are point sources of Rhodococcus. Moreover, none of the collected strains harbored known virulence genes, calling into question the role of these common environmental bacteria in causing PBTS
\end{abstract}

Keywords: microbial genomics, PBTS, pistachio, Rhodococcus

In 2011, defective clonal UCB1 pistachio rootstocks were detected in California orchards. Trees showed stunted shoots and stem galling, aberrant root architecture, and cracking around the bud union, which often led to graft failure. This condition was designated pistachio bushy top syndrome (PBTS) and attributed to two bacterial strains 
belonging to the genus Rhodococcus, assigned as $R$. fascians isolate 2 (PBTS2) and $R$. corynebacterioides-like isolate 1 (PBTS1) (Stamler et al. 2015). The postulate that PBTS1 and PBTS2 are responsible for disease was based on the following observations: (i) association of Rhodococcus bacteria with PBTS-affected orchards but not with nonaffected orchards; (ii) reduced plant growth following inoculation with high titers of PBTS2 and/or PBTS1; and (iii) polymerase chain reaction (PCR)-based detection of virulence genes in both PBTS2 and PBTS1. Based on this reasoning, the detection of Rhodococcus in pistachio nurseries and orchards led to the prophylactic replacement of more than one million pistachio trees to mitigate the spread of PBTS (Chang et al. 2018; Savory et al. 2017; Stamler et al. 2015).

The genus Rhodococcus belongs to the gram-positive phylum Actinobacteria, and its representatives are found in myriad environments. Among the diverse Rhodococcus species, only $R$. fascians and related members of the $R$. fascians "assemblage" are phytopathogens (Creason et al. 2014a; Sangal et al. 2019; Savory et al. 2017). Primarily known as a pathogen of herbaceous perennials, $R$. fascians causes leafy gall syndrome characterized by a loss of apical dominance, reduced senescence, and the formation of multiple inflorescences that cause the host to adopt a stunted, bushy appearance (Putnam and Miller 2007; Stes et al. 2013). $R$. fascians pathogenicity requires the virulence-associated genes most commonly observed to be embedded within a 200-kb conjugative linear plasmid, pFiD188. The well-characterized $R$. fascians strain D188 does not cause disease in the absence of pFiD188, whereas introducing this plasmid into nonpathogenic Rhodococcus isolates confers the ability to induce fasciation symptoms in diverse plant species (Crespi et al. 1992; Savory et al. 2017; Stes et al. 2013).

The taxonomy of the genus Rhodococcus has undergone numerous revisions since its initial description (Francis and Vereecke 2019). The initial classifications were based on disease symptoms, cell morphology, and genetic and biochemical characteristics and have since been found not to represent the evolutionary relationships inferred from genomic data. Genomebased taxonomies have indicated that the $R$. fascians assemblage is likely a distinct genus with multiple species (Creason et al. 2014a; Sangal et al. 2019; Savory et al. 2017). A phylogenomic study by Savory et al. (2017) revealed that $R$. fascians is part of a larger group of plant-associated Rhodococcus that can be divided into four well-resolved clades (I to IV). The Rhodococcus isolates within these four clades are further partitioned into 17 individual species based on pairwise average nucleotide identity (ANI) values exceeding $95 \%$ between genomes, the commonly accepted threshold for strains that are the same species (Richter and Rossello-Mora 2009). Fourteen of those species are known to associate with plants and contain both pathogenic and nonpathogenic strains. Eight of the 14 have been found to carry a linear plasmid with associated virulence loci (Savory et al. 2017).

The conclusion that $R$. fascians causes PBTS has been challenged by groups unable to replicate the Rhodococcus-associated growth inhibition of pistachio trees as reported by Stamler et al. (2015) and Savory et al. (2017). This debate has recently expanded to almond trees, as Rhodococcus spp. were found colonizing almond trees manifesting symptoms commonly associated with PBTS (Dhaouadi et al. 2021). The conflicting data and interpretation have led to a debate in the literature as to whether $R$. fascians causes PBTS and how the detection of Rhodococcus sp. in PBTS-afflicted pistachio orchards should guide the response of pistachio growers (Chang et al. 2018; Randall et al. 2018; Vereecke 2018; Vereecke et al. 2020). Given the substantial implications for both the pistachio producers and the nurseries that supply them, it is essential to determine what role, if any, Rhodococcus species play in PBTS. A clearer understanding of the relationship of Rhodococcus populations in nurseries, orchards, and wild environments could provide insight into the association between Rhodococcus and the recent PBTS outbreaks.

The ability to distinguish bacterial lineages is essential to describe bacterial population dynamics, evolution, and epidemiology. Here, we implemented a phylogenomic approach to characterize a collection of bacterial isolates sampled from symptomatic and asymptomatic pistachio trees from both pistachio nurseries and orchards. Additionally, we sampled a set of perennial and woody plant species from agricultural and riparian areas. Core gene phylogenies, ANI, single nucleotide polymorphism (SNP), and pangenome analyses assigned each isolate to a specific taxonomic group. These data elucidate Rhodococcus spp. distribution relative to host plant species and permit comparison of Rhodococcus communities within and among pistachio production areas, nurseries, and adjacent wild environments. Our findings indicate that several distinct Rhodococcus species, including $R$. fascians, $R$. kroppenstedtii, $R$. corynebacterioides, and several unnamed species, colonize diverse flora both within and around pistachio production areas in the San Joaquin Valley. Importantly, the presence of $R$. fascians, $R$. kroppenstedtii, and $R$. corynebacterioides, which have been implicated as species with members being causative agents of PBTS, did not predict the occurrence of PBTS. Additionally, the data fail to link Rhodococcus communities in orchards with the nurseries from which trees were derived. Moreover, none of the isolate's genomes contain evidence of either the linear plasmid or virulence genes commonly understood to be required for pathogenicity.

\section{MATERIALS AND METHODS}

\section{Surveys and sample collection}

An initial survey was conducted during the 2015 growing season to investigate the occurrence of Rhodococcus spp. in pistachio trees and other woody perennials in California. Sampling included symptomatic and asymptomatic pistachio trees in PBTS-affected orchards planted between 2011 and 2014 (Outbreak 1), as well as healthy pistachio trees and other fruit and nut trees in orchards planted prior to 2011. In addition, pistachio saplings and other fruit and nut tree-planting materials occurring on benches and greenhouses at three nurseries were sampled. Finally, sampling also included mature, asymptomatic ornamental and native plant species occurring in urban landscapes and riparian areas in California, respectively.

A second survey was conducted during the late summer and fall of 2016 following a second outbreak (Outbreak 2) of PBTS in pistachio orchards planted between 2015 and 2016 and with trees originating from a second nursery (Nursery 2). Sampling included symptomatic and asymptomatic trees in PBTS orchards, as well as at the nursery (Nursery 2). See Supplemental Methods for additional information.

Plant parts sampled included leaves of clonal UCB1 rootstock trees (symptomatic and asymptomatic) and bark tissues near galls of clonal UCB 1 pistachio rootstocks expressing PBTS symptoms. Additional tissues sampled included leaves from unaffected pistachio scions grafted onto symptomatic or asymptomatic clonal UCB1 rootstocks. Three to four leaves per tree, including old and young leaves, were collected using gloved hands with gloves that were changed between each sample. Individual samples were placed into plastic zippered storage bags and kept in a cooler during travel between the field and the laboratory. 
Leaf and bark samples were processed in the laboratory within 12 to $48 \mathrm{~h}$ following field collection. Isolation of Rhodococcus bacteria from the various tissues was done as described by Stamler et al. (2015). The adaxial and abaxial leaf sides were printed onto D2 agar media (Kado and Heskett 1970), and leaf imprints were taken by firmly pressing the surface of leaves onto Petri plates containing D2 media using a sterile glass rod. Internal and external bark tissues were also printed onto Petri dishes containing D2 medium. New nitrile disposable gloves were used between each sample.

To detect putative endophytic Rhodococcus bacteria, additional leaf and bark tissues were surface-sterilized in $0.6 \%$ sodium hypochlorite ( $40 \mathrm{~s}$ for leaves, 2 min for bark), washed with $70 \%$ alcohol, and rinsed twice with sterile water before being homogenized in $1 \times$ phosphate-buffered saline (PBS). Two-hundred microliters of the resulting homogenate were plated onto D2 agar media and incubated at $28^{\circ} \mathrm{C}$ for up to 14 days to allow bacterial colonies to grow. Petri dishes were checked every 2 days for the occurrence of yellow to orange-red bacterial colonies (Stamler et al. 2015). Single colonies characteristic of Rhodococcus bacteria were selected from the surface of the medium and restreaked twice onto new D2 medium Petri dishes to obtain pure cultures.

\section{S rRNA gene sequencing}

Pure colonies were transferred into sterile Eppendorf tubes containing a lysing matrix, and the total genomic DNA was extracted with a FastDNA Spin Kit (MP Biomedicals, Santa Ana, CA). Preliminary identification of bacterial isolates was done following amplification and sequencing of the 16S rRNA gene using the primers and PCR conditions reported by Nikolaeva et al. (2012).

\section{Nucleic acid preparation}

Rhodococcus isolates were maintained on solid lysogeny broth (LB) medium at $28^{\circ} \mathrm{C}$ or grown overnight in $\mathrm{LB}$ medium at $28^{\circ} \mathrm{C}$ with shaking for DNA extraction. The Qiagen DNeasy Blood and Tissue Kit was used for bacterial DNA extraction following the recommended protocol for the lysis of gram-positive bacterial cells. Briefly, cell pellets were resuspended in $180 \mathrm{ul}$ of an ethylenediaminetetraacetic acid (EDTA)-enzyme-detergent solution (20 mM of TrisCl, pH 8.0, $2 \mathrm{mM}$ of sodium EDTA, 1.2\% Triton X-100 and lysozyme to $20 \mathrm{mg} / \mathrm{ml}$ ) and incubated at $37^{\circ} \mathrm{C}$ for $90 \mathrm{~min}$. The proteinase $\mathrm{K}$ lysis step was extended to $90 \mathrm{~min}$ in Buffer $\mathrm{AL}$ at $56^{\circ} \mathrm{C}$ ( $3 \mathrm{~h}$ total for both enzymatic incubations) to increase DNA yield (as recommended by the manufacturer). Methods for binding, washing, and eluting in the Qiagen spin columns were performed as recommended.

\section{Genome sequencing}

Genome library construction and sequencing were performed using either the Illumina Nextera XT library preparation kit or the QiaSeqFX. The barcoded libraries, size selected to contain about 600- to 700-bp inserts, were pooled and sequenced on the HiSeq4000 using a 150-bp paired-end protocol. Illumina quality control was conducted using Trimmomatic v3.6 (Bolger et al. 2014), and genome assemblies were performed using the SPAdes v3.13 assembly package with the parameter "k 21,33,55,77,99,127 -careful" (Bankevich et al. 2012). Genome completeness was assessed using BUSCO v4.1.4 with the "bacteria_odb10" dataset (Waterhouse et al. 2018).
Bacterial genome annotation on both BP and reference genomes was performed using Prokka (Seemann 2014), and phylogenetic analysis was conducted on the Prokka output using PhyloPhlAn v.3.0. PhyloPhlAn is an integrated pipeline that identifies and utilizes 400 core proteins to construct phylogenetic trees (Segata et al. 2013). Additionally, SNPs were called by GATK (version gatk-4.2.1.0) (Van der Auwera and O'Connor 2020). Rhodococcus sp., Leaf 225 , and $R$. fascians D188 were used as reference genomes to identify SNPs in Rhodococcus sp. (Clade IIIb) and $R$. fascians genomes, respectively. A custom script was used to convert the vcf file to fasta format for phylogenetic analysis. Phylogenetic analysis was performed using BEASTv1.10.4 (Drummond et al. 2012) with default parameters except for the tree prior model:Yule process. The Newick (PhyloPhlAn) or Nexus (BEAST) output files were visualized and annotated using the online tools in iTOLv6 (https://itol.embl.de/).

Average nucleotide identity and Hadamard calculations were done using the "MUMMER" option in PyANI (Pritchard et al. 2016). Heatmaps were annotated using the "pheatmap" package in R (pheatmap v.1.0.12). Genome alignments were performed with Progressive Mauve (Darling et al. 2004), and maps were produced using Microreact (Argimon et al. 2016).

For non-Rhodococcus taxa, reference genomes were selected by querying GenomesDB using the Tetra Correlation Search (TCS) in the JSpeciesWS web-based tool (http: //jspecies.ribohost.com/jspeciesws/) to identify closely related genomes (Richter and Rossello-Mora 2009). Pangenome analysis was conducted using Roary (v. 3.11.2) (Page et al. 2015) and visualized using Phandango (https://jameshadfield.github.io/ phandango/\#/).

Illumina reads were mapped to the $\mathrm{pFiD} 188$ linear virulence plasmid (Crespi et al. 1992) using BWA ALN 0.7.9a-r78640 (Li and Durbin 2009), requiring end-to-end mapping while allowing 10 mismatches per 150-bp read. Reads were filtered using SAMtools (Li et al. 2009) to account for PCR duplicates, and the number of unique cluster fragments were counted for each sample.

\section{RESULTS}

Over the past decade, two PBTS outbreaks have occurred in California, for which two nurseries (Nursery 1 and Nursery 2) were implicated as point sources for defective rootstocks and suspected pathogens. Outbreak 1 occurred on clonal UCB 1 trees planted between 2011 and 2014 and was associated with Nursery 1. Outbreak 2 occurred on clonal UCB1 trees planted from 2015 to 2016 and was associated with Nursery 2. Symptomatic clonal UCB1 trees showed mild to severe cracking of the bark below the graft union, swelling of the bark with gall-like structures in underlying wood tissue, excessive branching, and outgrown bark lenticels producing a speckled pattern on the trunk of rootstocks. To characterize and compare the Rhodococcus populations within these nurseries and the orchards they supplied, we sampled bacteria from leaves of symptomatic and asymptomatic pistachio trees from these locations. Isolates were also collected from symptomatic bark tissues to potentially enrich the causal agent of PBTS from those trees. Additional trees sampled during Outbreak 1 included stunted clonal UCB1 rootstocks with short internodes. This growth abnormality imparted a characteristic bushy appearance to the affected trees but was not accompanied by cracks or galls. To further examine the incidence of Rhodococcus and its relationship to host status, we sampled pistachio orchards with no evidence of PBTS, as well as plant species other than pistachios 
from three different nurseries, orchards, and various native and ornamental woody species. None of the alternative species we tested exhibited PBTS-like symptoms.

Candidate Rhodococcus colonies were selected based on colony morphology and color (Stamler et al. 2015), and preliminary identification was performed using 16S rRNA amplicon sequencing (Nikolaeva et al. 2012). A complete accounting of all isolates is presented in Materials and Methods and summarized in Supplementary Table S1. Isolates were labeled with the prefix BP ("Bacterium from Pistachio").

\section{Symptomatic and asymptomatic pistachio trees harbor a diverse population of Rhodococcus species}

As a first step to understand the relationship between Rhodococcus spp. and PBTS, we sequenced the genomes of 231 isolates and delineated their species identities and genetic relatedness. Genomes were sequenced and assembled as described in Materials and Methods with the assembly metrics presented in Supplementary Table S1. We implemented the PhyloPhlAn integrated pipeline to construct a phylogenetic tree from an alignment of more than 400 core protein sequences (Segata et al. 2013). This analysis elucidated the taxonomic relationships of the isolates relative to 39 reference strains: 33 Rhodococcus strains representing the 17 Rhodococcus species clades (Savory et al. 2017) and 6 representing additional related strains and type strains (T). Nocardia farcinica was used as an outgroup to root the tree.

The majority of the collection was partitioned into three clades accompanied by several less populated outgroups (Fig. 1). Membership in these clades was supported by ANI comparisons such that the genomes of isolates within each clade shared ANI values greater than $95 \%$, the threshold for membership in the same species (Fig. 2). The largest clade (Fig. 1, Red clade) contained 150 isolates that were co-phyletic with known pathogenic $R$. fascians isolates including D188 and other $R$. fascians isolates assigned to Group Ia in Savory et al. (2017). Also belonging to this clade is PBTS2. Representatives in this clade were isolated during both Outbreak 1 and Outbreak 2 and were also found on trees planted prior to either outbreak. Reference strains representing the other Type I species groups from Savory et al. (2017) (Type I $\mathrm{b}$ to $\mathrm{h}$ ) all formed an outgroup to the $R$. fascians Type Ia strains in our collection. These findings indicate that a single Rhodococcus species, $R$. fascians Type Ia, dominated our collection.

Several smaller clades contain the remaining strains in the collection. A clade containing 31 isolates (Fig. 1, Orange clade) partitioned with the Rhodococcus reference strain $R$. kyotonensis KB10 (Hong et al. 2015), representing Clade IId from Savory et al. (2017). The genomes of these 31 isolates share over $99.99 \%$ ANI with each other and over $98 \%$ ANI with $R$. kyotonensis $\mathrm{KB} 10$, indicating their conspecific nature (Supplementary Table S2). However, the R. kyotonensis-type strain JCM23211 [T] (Li et al. 2007) shares only about 85\% ANI with KB10 and the 31 isolates from this new collection, indicating that they represent a novel Rhodococcus species, hereafter referred to as Rhodococcus sp. Clade IId. Rhodococcus KB10 was isolated from Arabidopsis thaliana and is described as an endophyte (Hong et al. 2015). The related Rhodococcus sp. Clade IId strains in our collection were isolated almost exclusively from UCB1 leaves during the second PBTS outbreak (Fig. 1). There are no reports of Rhodococcus sp. Clade IId behaving as a plant pathogen, nor of it possessing the linear virulence plasmid as does $R$. fascians (Savory et al. 2017).

A third clade contained 37 isolates (Fig. 1, Yellow clade) synonymous with Rhodococcus Clade IIIb (Savory et al. 2017). Clade IIIb contains Rhodococcus sp. "Leaf 225," which was iso- lated from Arabidopsis leaves (Bai et al. 2015). Although members of Clade IIIb represent a single species (ANI $>95 \%$, Fig. 2), the clade is further divisible into subgroups based on ANI and the Hadamard metric (ANI $\times \%$ genome alignment; Fig. 2, Supplementary Fig. S1) and is further supported by a phylogenetic tree constructed using whole genome SNP analyses (Fig. 3A, see below). Strains assigned to Clade IIIb subgroup A (Fig. 3A) were isolated during the second PBTS outbreak and were collected primarily from Nursery 2, with a single isolate from Nursery 3 . These strains share pairwise ANI and coverage values near $100 \%$ (Supplementary Fig. S1, Supplementary Table S2). Based on whole genome alignment values, strains assigned to Clade IIIb subgroup B (Fig. 3A) also have highly similar genome content but have diverse origins - collected at different times from different locations and plant species (Fig. 1, Supplementary Table S1). Three additional strains are basal to the subgroup B strains but more diverged.

Four strains represent Clade IVb and are co-phyletic with the previously reported pathogenic strain PBTS1 (Fig. 1, Green clade) (Savory et al. 2017). PBTS1 was originally reported as $R$. corynebacterioides-like based on $16 \mathrm{~S}$ sequence (Stamler et al. 2015). A TCS of the GenomesDB database with PBTS1 and the co-phyletic genomes indicated that the most related genome in the database is R. kroppenstedtii 44908 [T] (Mayilraj et al. 2006). Pairwise ANI analysis revealed that the genomes from PBTS1 and the other Clade IVb strains isolated in this study are over $97 \%$ identical to R. kroppenstedtii 44908 [T] and less similar to $R$. corynebacterioides NBRC14404 [T] (<92\%) (Fig. 1). Thus, PBTS1 is conspecific with $R$. kroppenstedtii and distinct from $R$. corynebacterioides. Two additional strains, BP-281 and BP-282 (Fig. 1, Magenta clade), were similar to R. kroppenstedtii 44908[T], but their ANI falls below the 95\% threshold, indicating that they represent a new Rhodococcus species.

Clade IVa (Fig. 1, Blue clade) contains $R$. corynebacterioides NBRC14404[T], as well as two isolates from our collection, BP-287 and BP-317. ANI values again support the phylogenetic analysis and confirm that BP-287 and BP-317 are R. corynebacterioides. The remaining samples in our collection were not of the genus Rhodococcus but instead represented two distinct Gordonia sp. and a single isolate of Dietzia cinnamea; as expected, these genomes occurred as outgroups in the phylogenomic tree (Fig. 1).

\section{R. fascians is not restricted to symptomatic samples or orchards}

Among all species identified in our collection, only $R$. fascians (Fig. 1, Clade Ia) and $R$. kroppenstedtii (formerly $R$. corynebacterioides-like, Fig. 1, Clade IVb) have been implicated in causing PBTS (Stamler et al. 2015). We were interested to know if either of these species was more often associated with pistachio samples expressing PBTS symptoms. We annotated Figure 1 to denote which strains were isolated from trees exhibiting PBTS symptoms. Disease symptoms were not correlated with phylogenetic placement, and indeed, all clades are a mosaic of strains originating from symptomatic and asymptomatic trees. Of particular interest are the many (73/153) $R$. fascians-type Ia isolates cultured from asymptomatic trees, including numerous strains originating from mature pistachio trees planted years before either PBTS outbreak or from species other than pistachio. Three of the four $R$. kroppenstedtii isolates were derived from the bark of symptomatic UCB1 pistachio trees (the fourth isolate came from an asymptomatic walnut tree), whereas only one of the two $R$. corynebacterioides strains originated from the tissue of symptomatic trees. The infrequent occurrence of 
$R$. corynebacterioides and $R$. kroppenstedtii precludes inferences regarding their association with symptomatic or asymptomatic trees.

\section{R. fascians contains several distinct genotypes based on SNPs, genome alignments, and gene content}

The current collection is dominated by $R$. fascians isolates related to those in Clade Ia of Savory et al. (2017), which also includes PBTS2, one of the isolates implicated as a causal agent for PBTS. We therefore examined the genetic diversity and distribution of this subset of isolates using SNP and ANI analyses. As shown in Figure 3B, R. fascians further partitions into several lineages that we designate Groups A to K. Five of these subgroups had at least five members, whereas the majority of the collection occurred in a single large clade containing 83 isolates (subgroup A). The next largest clades had 18, 17, 11, and 5 representative isolates with several outgroups consisting of only 1 or 2 isolates.

Pairwise ANI values among all $R$. fascians genomes in Clade Ia were over $97 \%$ (Supplementary Table S3), well above the generally accepted $95 \%$ cutoff for bacterial species (Richter and Rossello-Mora 2009). Despite high ANI values, the percentage of any two genomes that aligned was variable among groups, ranging from 78 to $100 \%$ (Supplementary Table S3). The hierarchal heatmap generated from the Hadamard data (Fig. 4, Supplementary Table S3) divides the species into distinct subsets of highly similar groups of strains congruent with SNP analyses (Fig. 3B). Because these groups differ primarily in the percentage

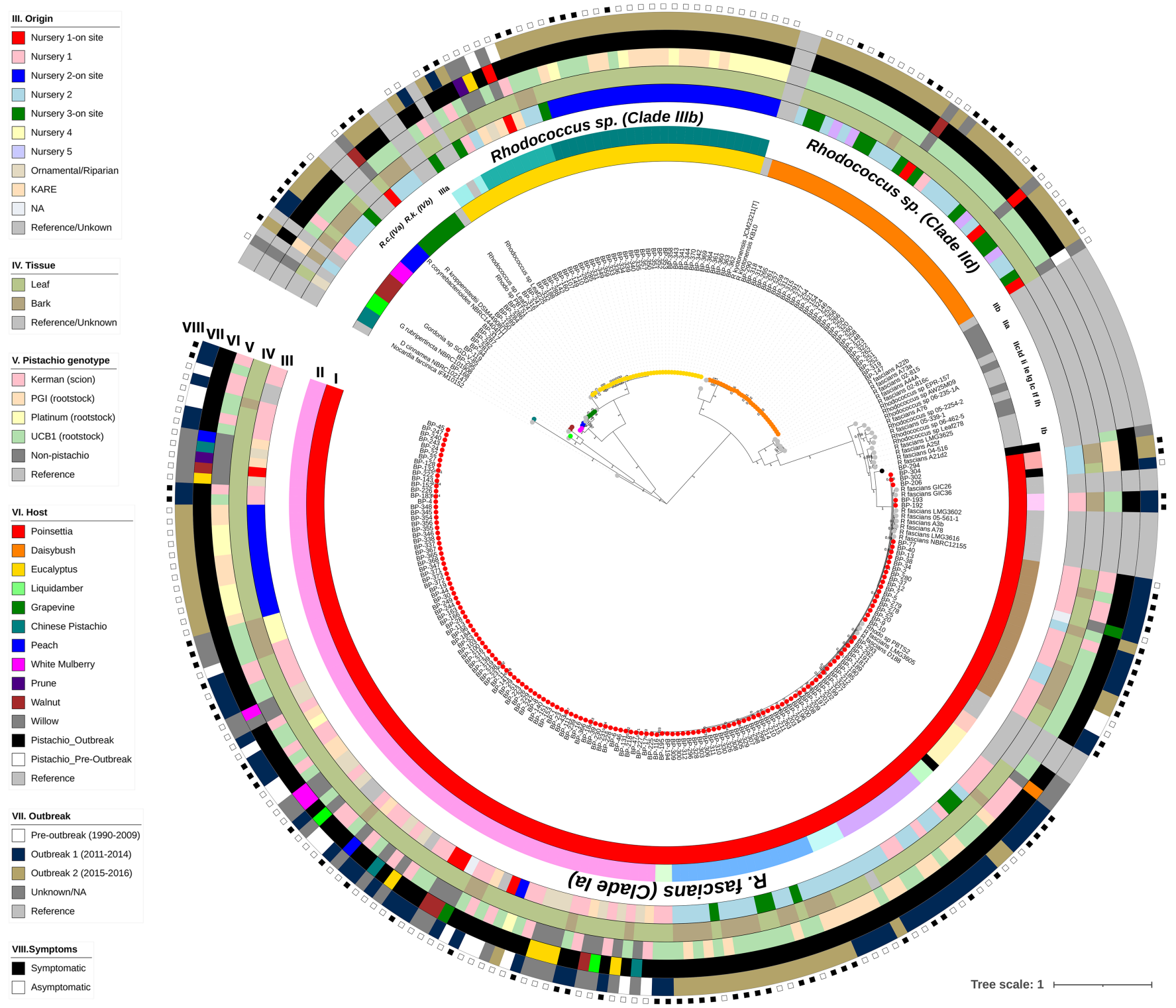

FIGURE 1

Phylogenomic analysis indicates that our collection encompasses at least six Rhodococcus species, including the plant pathogen R. fascians. Phylogenetic tree constructed with PhyloPhIAn (Segata et al. 2013). Each concentric ring is colored to reflect the following metadata: I = strains sharing $>95 \%$ average nucleotide identity, which are by definition a common species; $I I=$ subgroups of species determined by single nucleotide polymorphism analysis, gene content, and genome alignments (see also Fig. 3); III = the location from which strains were isolated; IV = the tissue from which strains were isolated; $V=$ the genotype of the pistachio host; $\mathrm{VI}=$ the plant species from which the strains were isolated; $\mathrm{VII}=$ the outbreak during which the strain was isolated; and VIII = whether the isolate came from symptomatic or asymptomatic tissue. The significance of each color in each ring is presented in the key on the left. Clades are labeled according to Savory et al. (2017) using the reference strains described therein. Bootstrap values $>0.70$ are indicated on the branches. 
of alignable genomes, they are likely to have arisen by the acquisition or loss of chromosomal segments or extrachromosomal replicons (see below).

To understand the extent and dynamics of the $R$. fascians pangenome, each individual genome was annotated, and their gene contents were compared (Page et al. 2015). We identified, on average, 5,054 genes in the $R$. fascians genomes (range 4,927 to 5,725 genes), similar to the 5,475 coding sequences (CDSs) reported by Creason et al. (2014b). A hierarchically clustered gene presence-absence matrix (Fig. 5) partitions the strains into the same groups as did the SNP or Hadamard analyses, indicative of multiple conserved sublineages that are divergent from one another for gene content. Considering the 150 strains collected here and 11 reference genomes, we identified a core genome (genes present in $>99 \%$ of strains) of 3,589 genes and a total pangenome of 15,131 . Among the reference strains, only PBTS2, previously isolated from pistachio, and $R$. fascians strain 05-5611, a nonpathogenic strain isolated from Lavandula angustifolia in Washington, U.S.A. (Serdani et al. 2013; Stamler et al. 2015), were co-phyletic with the new strains characterized here. In
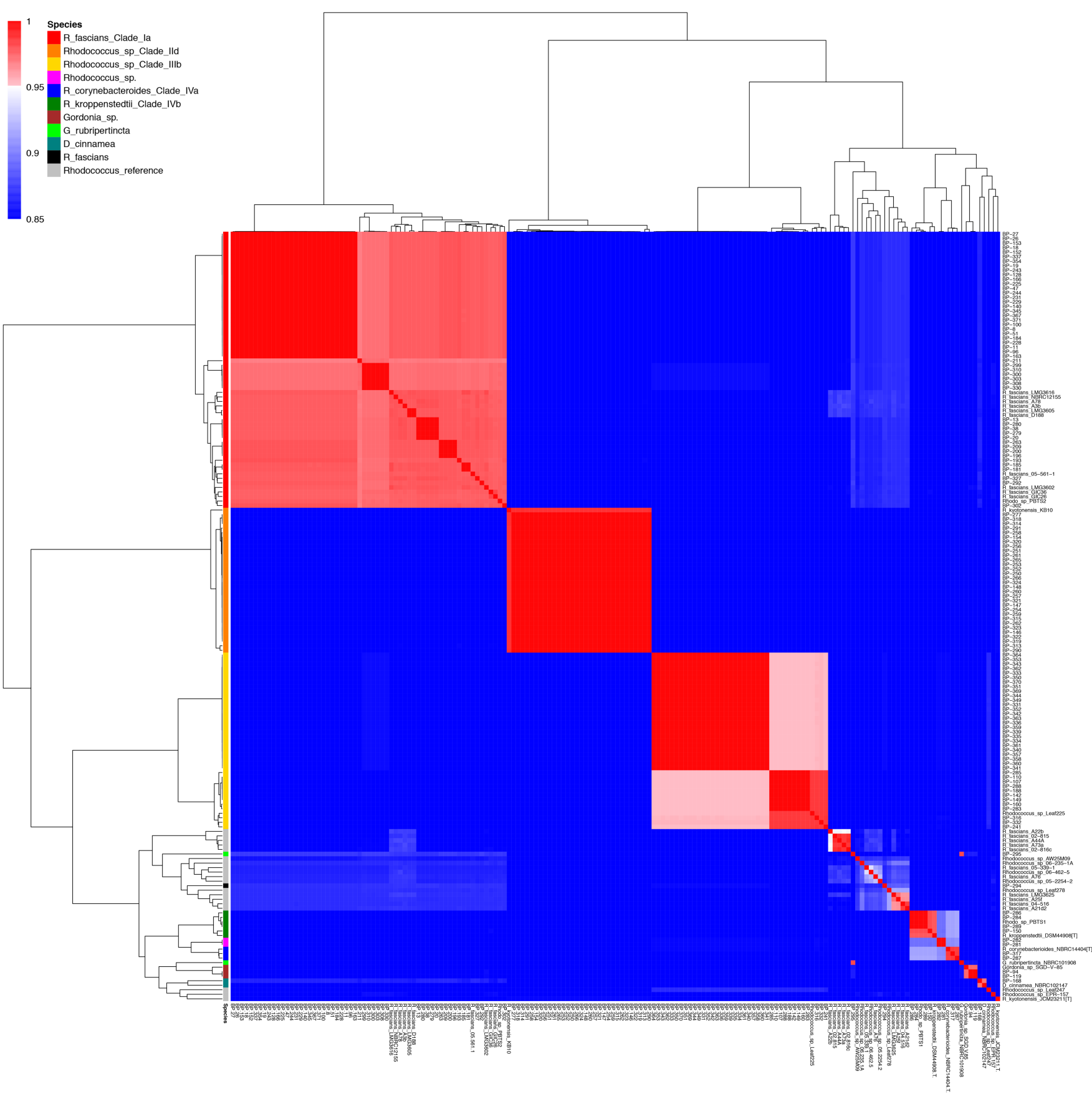

FIGURE 2

The genomes from isolates that are cophyletic in the PhyloPhIAn tree share $>95 \%$ average nucleotide identity (ANI) and represent a common species. ANI values were calculated using PyANI and presented as a heatmap. Color gradient denotes the percent identity values, with all ANI scores $>95 \%$ (the species threshold) colored pink to red and values $<95 \%$ shaded blue. The hierarchical tree was constructed based on the ANI output, with high values clustered together. A representative sample of the Rhodococcus fascians genomes was selected to facilitate the analyses. Species groups are color coded to the left of the heatmap according to the legend and correspond to the colors of the phylogenetic tree leaves in Figure 1. 
summary, SNP, ANI, and pangenome comparisons all define the same groups of closely related isolates.

\section{Rhodococcus diversity in pistachio nurseries does not reflect diversity in orchards}

Since 2011, the two PBTS outbreaks were attributed to two nurseries allegedly distributing Rhodococcus-infected trees to growers. Outbreak 1 (2011 to 2014) was attributed to Nursery 1, and Outbreak 2 (2015 to 2016) was attributed to Nursery 2. Diverse Rhodococcus isolates were collected from sites spanning our sampling range with multiple species and genome types identified from individual nurseries and orchards (Supplementary Fig. S2, Supplementary Table S1). If the nurseries were the source of Rhodococcus found in orchards, we predicted that related lineages with recent common ancestry would reside in both the orchards and the nurseries from which the pistachio trees were obtained. We compared the isolates in our collection that originated in nurseries and orchards during the two outbreaks to examine this hypothesis.

Nine Rhodococcus isolates were retrieved from leaf samples taken from asymptomatic plants in Nursery 1 greenhouses, including nut trees and an ornamental shrub (Supplementary Table $\mathrm{S} 1)$. Among these isolates were four $R$. fascians Group A, three Rhodococcus sp. Clade IId, and one each of Rhodococcus sp. Clade IIIb Group B and R. kroppenstedtii (Fig. 6, Nursery 1-On site). We compared the strains identified on site at Nursery 1 with the strains isolated between 2009 and 2014 from orchard trees that were purchased from Nursery 1 (Fig. 6, Nursery 1-Orchards). Fifty-nine isolates were obtained from 47 trees, among which
95\% (56 of 59) were R. fascians. A majority (31 of 56) were $R$. fascians Group A (Fig. 3B), a closely related group that was also present on site at Nursery 1. However, R. fascians Group A was ubiquitous and frequently found on trees originating from Nurseries 2 and 4 between 2009 and 2014 (Fig. 1, Supplementary Table S1), neither of which was implicated as a source of PBTS during Outbreak 1. Furthermore, $R$. fascians Group A was also isolated from a variety of non-pistachio host plants (Fig. 1). Conversely, Nursery 1-derived clones in orchards harbored additional $R$. fascians groups, none of which was identified on site at Nursery 1, including Groups D (13), E (4), F (4), G (2), and I (2) (Fig. 6, Nursery 1).

Nursery 2 was implicated as the source of the 2015 to 2016 outbreak. We sampled symptomatic UCB1 rootstock trees and asymptomatic pistachio clones "PGI" and "Platinum" from Nursery 2 and from orchards supplied by Nursery 2 . In total, 38 Rhodococcus strains were obtained from 12 trees at Nursery 2, representing 25 Rhodococcus $\mathrm{sp}$. Clade IIIb strains and 13 strains that were R. fascians Group A (Fig. 6, Nursery 2-On site). Among four strains obtained from symptomatic UCB 1 trees at Nursery 2, all were Rhodococcus sp. Clade IIIb. Ten asymptomatic pistachio trees, with equal representation of PGI and Platinum clones, yielded a mixture of $R$. fascians subgroup A and Rhodococcus sp. Clade IIIb from their leaves. In contrast, sampling strains from pistachio trees in orchards sourced from Nursery 2 stocks (Fig. 6, Nursery 2-Orchards) yielded a completely different set of 42 Rhodococcus genotypes. R. fascians and Rhodococcus sp. Clade IId were the predominant species isolated from the symptomatic trees, representing 48 and $28 \%$ of isolates, respectively. The $R$. fascians strains represented four different Groups: B, D, E, and
A

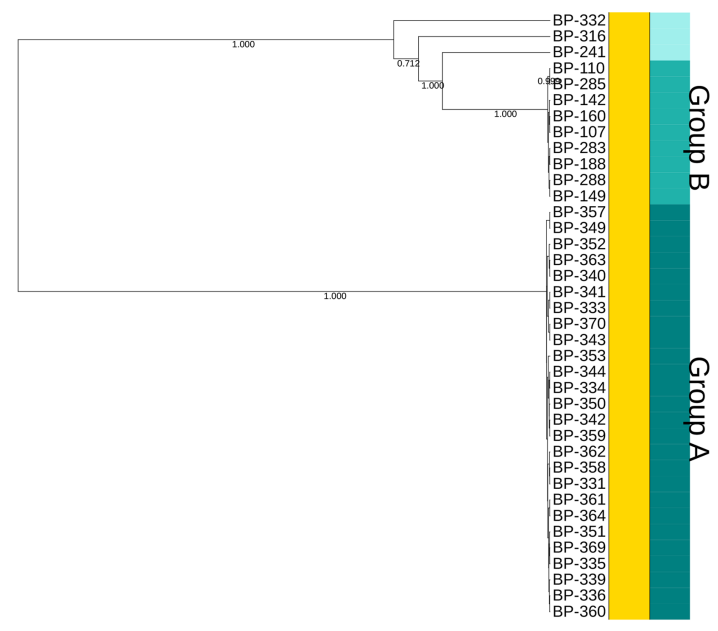

B

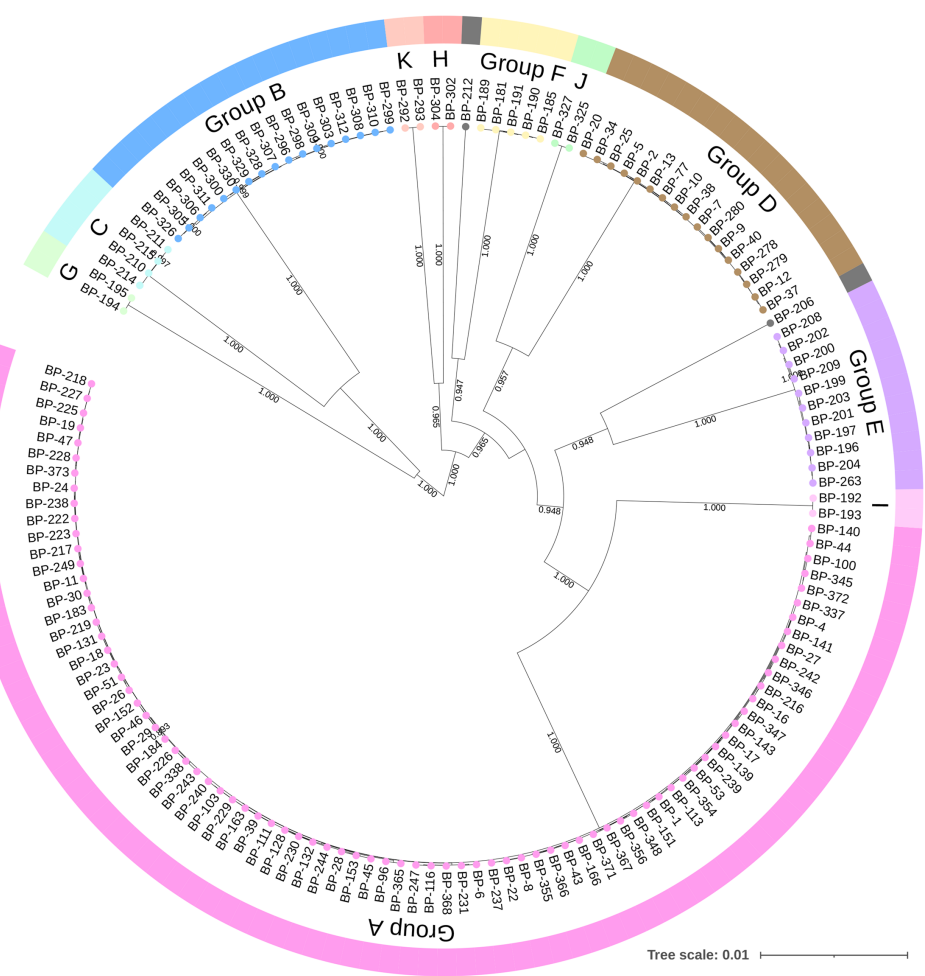

FIGURE 3

Single nucleotide polymorphism (SNP) analysis further differentiates species into genetically distinct subgroups. Species-specific phylogenetic trees constructed for Rhodococcus sp. (IIIb) and R. fascians. A, Rhodococcus sp. (IIlb) genomes were compared with the Rhodococcus sp. Leaf225 reference, and 10,257 SNPs were identified and used to create the tree. Individuals within Groups A and B could not be differentiated, and BP-316, BP-241, and BP-332 are not as closely related to each other as the genomes in Group A and Group B are to each other. B, Comparing $R$. fascians genomes to the $R$. fascians D188 reference reveals 18,319 SNPs that define 11 genetically distinct subgroups. The groups identified in both trees are consistent with Hadamard values (ANI $\times \%$ genome overlap, Fig. 4) and pangenome analysis (Fig. 5). Branch labels denote posterior probabilities. 
$\mathrm{H}$, notably lacking representation from Group A, the dominant genotype found in Nursery 2. The remaining seven isolates from symptomatic trees belonged to diverse Rhodococcus species (Fig. 1, Fig. 6, Nursery 2-Orchards). These data indicate that Rhodococcus communities in Nursery 2-derived orchards differ from those at Nursery 2.

To further investigate the occurrence and diversity of Rhodococcus among California's pistachio nurseries and orchards, we sampled pistachio trees from nurseries not associated with either outbreak, including trees on site at Nursery 3 and orchard trees originating from two other nurseries, Nursery 4 and Nursery 5. Among isolates obtained from four asymptomatic trees at Nursery 3, Rhodococcus sp. Clade IId accounted for $55 \%$ of strains, whereas R. fascians, Rhodococcus Clade IIIb, and $R$. corynebacterioides accounted for 30,15 , and $5 \%$, respectively. Nursery 4-sourced trees sampled from orchards were all symptomatic and yielded exclusively $R$. fascians Group A isolates, whereas three healthy Nursery 5 trees in orchards yielded exclusively Rhodococcus sp. Clade IId. Taken together, these surveys of trees associated with Nurseries 3, 4, and 5 indicate that although $R$. fascians was occasionally found on symptomatic trees, it was equally likely to be found on asymptomatic trees and was also present in or on trees derived from nurseries with no known history of PBTS.

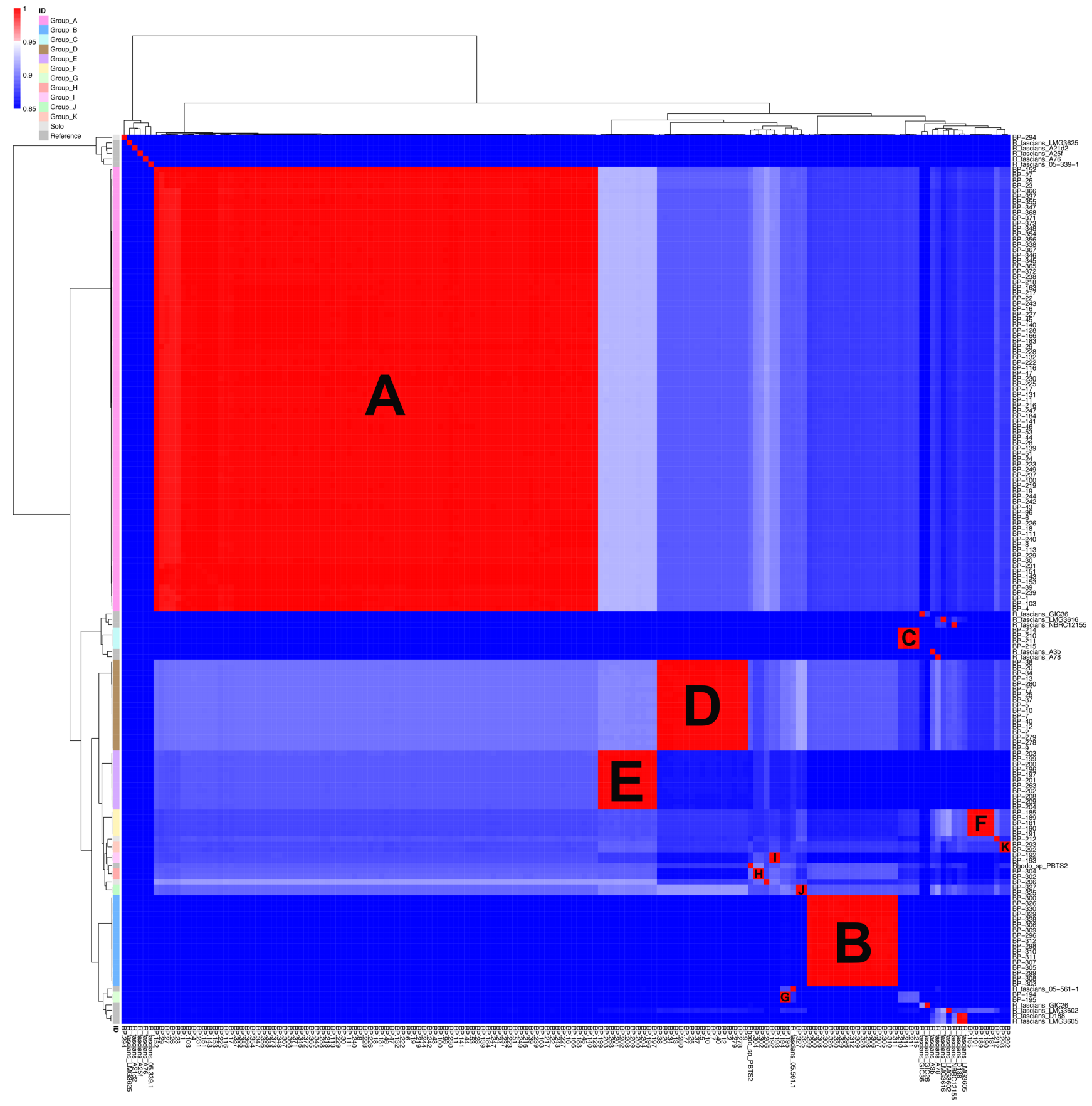

FIGURE 4

Hadamard analysis partitions strains into the same groups as single nucleotide polymorphism (SNP) analysis. Average nucleotide identity analysis of all strains in the Rhodococcus fascians clade was used to calculate Hadamard values (\% identity $\times \%$ overlap). An all-by-all comparison was performed, and hierarchical clustering was used to construct a heatmap. Groups with higher Hadamard values cluster together into groups which recapitulate clades deduced from phylogenomic SNP analysis. Groups are lettered and colored similar to Figure 3B. 


\section{Diverse Rhodococcus species populate flora outside of PBTS-affected areas}

The different composition of the Rhodococcus communities present in orchards versus nurseries suggests that nurseries are not the only source of Rhodococcus and that an endemic Rhodococcus population resides in the San Joaquin Valley. We examined this scenario by sampling native and cultivated tree species adjacent to pistachio orchards and nurseries. Among these were (i) mature, asymptomatic, woody plant species (walnut, peach, prune, and pistachio) at the Kearney Agricultural Research and Extension Center (KARE) in Parlier, CA; (ii) diverse woody flora from a riparian tree stand approximately $3.9 \mathrm{~km}$ from KARE (liquidambar, white mulberry, eucalyptus, and Chinese pistachio); and (iii) mature pistachio trees (Kerman scions) that were planted before the outbreak in 2000, none of which exhibited PBTS symptoms. Thirty-one Rhodococcus strains were obtained from these sites, including $28 R$. fascians isolates, all Group A, and 3 isolates of Rhodococcus sp. Clade IIIb subgroup B (Fig. 3A). This suggests that communities of $R$. fascians are endemic to woody flora external to pistachio orchards and that their strain membership is similar to that of pistachio nurseries and orchards, consistent with a local origin for $R$. fascians.

\section{Rhodococcus isolates from PBTS-afflicted trees lack the linear virulence plasmid}

The genome of the pathogenic $R$. fascians strain D188 has been fully sequenced (Francis et al. 2012; Stamler et al. 2016). Alignment of the D188 genome with genomes from strains representing $R$. fascians Groups A through F revealed broad conservation of the main chromosome, depicted as locally colinear blocks (LCBs) (Fig. 7). Several regions lacking homology to other geno- types were present, likely representing insertions or deletions. The D188 genome also contains two plasmids: a linear virulence plasmid required to cause disease (pFiD188) and a second, circular plasmid with no known role in virulence (pD188) (Crespi et al. 1992). Among $R$. fascians sampled and analyzed here, none contained scaffolds homologous to pFiD188. To control for the possibility that the virulence gene homologs did not assemble effectively in our genomes due to copy number or instability, we also mapped the full set of Illumina reads against the pFiD188 virulence plasmid, and with the exception of three cluster reads (among millions of total reads) of marginal identity and from a single sample, no reads mapped to the plasmid.

Prior genome sequencing studies suggested that a highly conserved linear plasmid resembling pFiD188 was present in diverse phytopathogenic Rhodococcus species (Creason et al. 2014b; Crespi et al. 1992; Savory et al. 2017). Such plasmids often contained the virulence genes responsible for eliciting disease. However, subsequent results demonstrated that the virulence genes also reside in atypical, circular plasmid backbones (Savory 2020). We used BLASTP to query both our sequenced genomes and $R$. fascians reference genomes using the FasA, FasD, and AttR protein sequences from $R$. fascians D188. The best scoring sequences identified in our collection shared less than $48 \%$ amino acid identity with D188 virulence proteins, well below the expected threshold for orthologous genes among related strains. By contrast, Creason et al. (2014a) found that virulence genes in the att, fas $R$, and fas loci are highly conserved among $R$. fascians. As a benchmark, proteins identified in the reference genomes of known phytopathogenic $R$. fascians strains were all more than 94 to $100 \%$ identical to the D188 virulence proteins (Supplementary Table S4). Thus, neither the virulence plasmid pFiD188 nor the characterized virulence genes of pathogenic $R$. fascians were detected in strains obtained from pistachio nurseries, orchards, and local ornamental or native plant species within the geographic range of PBTS outbreaks.

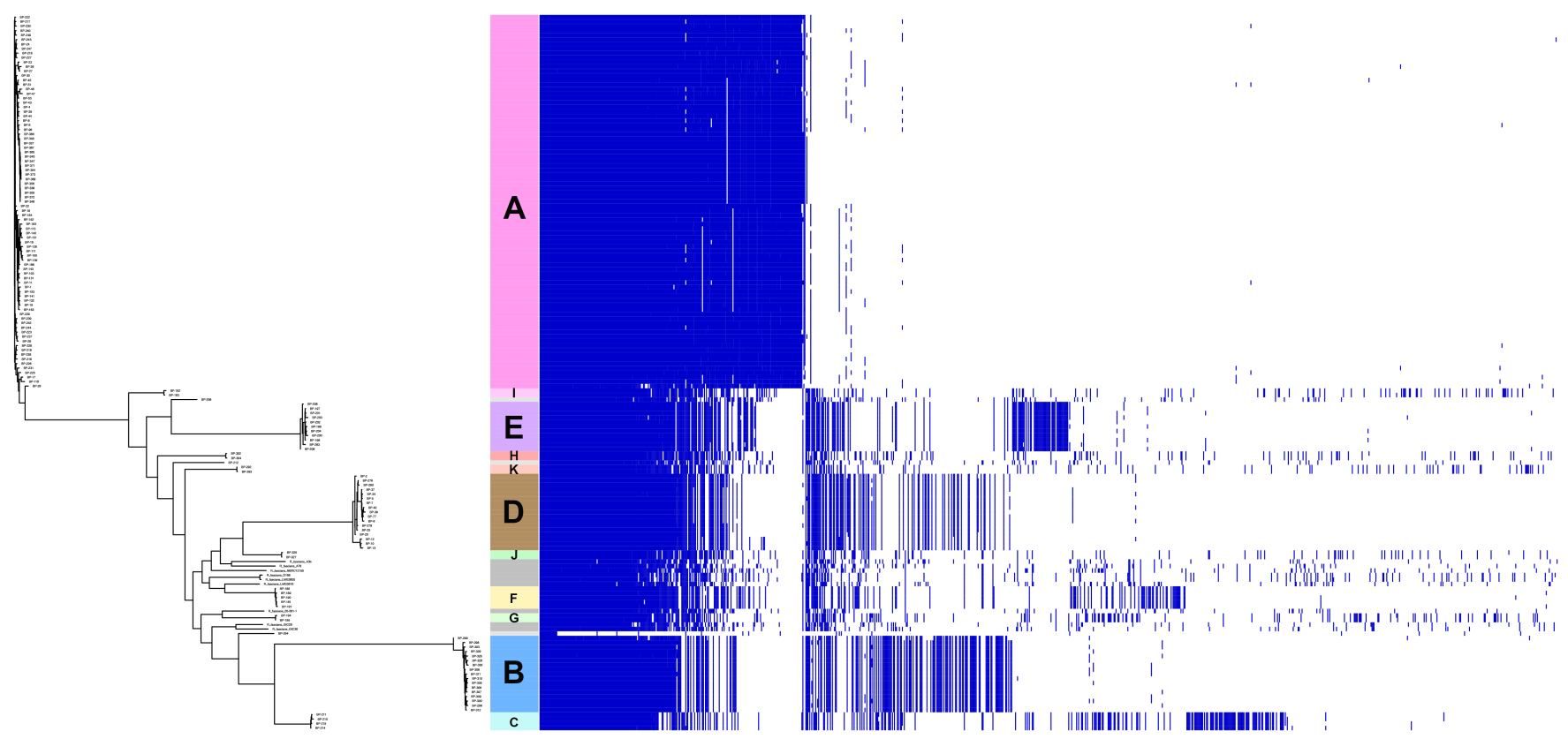

FIGURE 5

Pangenome analysis indicates that accessory genomes differentiate the Rhodococcus fascians genotypes. Hierarchical clustering of a $R$. fascians gene presence-absence matrix partitions the genomes into groups that correlate with those derived from phylogenomic and $\mathrm{ANI} /$ Hadamard analysis. Rows on the right denote the presence (blue) or absence (white) of genes in the genomes of strains indicated on the leaves of the adjacent tree (left). The groups are colored according to those used in Figure 3B, with reference genomes left white. 


\section{DISCUSSION}

\section{Rhodococcus is a cosmopolitan bacterial species populating diverse flora in both wild and agronomic environments}

PBTS has emerged as a serious problem for pistachio growers in the western United States, at the cost of lost productivity and the materials and labor to remove and replace afflicted trees. Two Rhodococcus species, including $R$. fascians, have been implicated as the causal agents of PBTS, although this claim remains controversial. To gain a better understanding of the genetic diversity of Rhodococcus populations in and around California's pistachio-producing areas, we collected Rhodococcus isolates from both symptomatic and asymptomatic pistachio trees and other woody plant species. We sequenced the genomes of the strains in our collection and used core gene phylogenetic analysis, ANI, and SNP analysis to elucidate the taxonomic relationships among the isolates, identifying at least six Rhodococcus species. Among the strains that were identified as $R$. fascians, only type Ia isolates were found, and these strains were genetically distinct from previously characterized $R$. fascians reference genomes, exhibiting dissimilarity in both genome sequence and gene content. These differences likely reflect the diverse geographical origins of the reference strains, which were isolated in different states and countries and from differing ecological contexts (Creason et al. 2014a). Although the majority of the PBTS-associated isolates were $R$. fascians, we found that Rhodococcus generally, and $R$. fascians specifically, is ubiquitous in and around the agricultural environment, often occurring epiphytically on both symptomatic and asymptomatic pistachio trees, as well as in other woody crops and native plant species.
Previous reports suggested that PBTS results from the activities of two different Rhodococcus species that co-occur on symptomatic pistachio trees (Stamler et al. 2015; Vereecke et al. 2020). In particular, isolates of $R$. fascians (PBTS2) and $R$. kroppenstedtii (PBTS1) have been reported to stunt plant growth when inoculated onto plants individually, and to greater effect in mixed inoculations. These two species were postulated to occupy distinct ecological niches, facilitating their co-occurrence (Vereecke et al. 2020). Notably, whereas $R$. fascians was frequently associated with the pistachio trees that we sampled, comprising $65 \%$ of all isolated strains, $R$. kroppenstedtii was significantly less common, and we did not observe coincidence of these two species on pistachio trees. We found only two plants that were colonized by $R$. kroppenstedtii: pistachio and walnut. Likewise, we identified two bona fide $R$. corynebacterioides colonies, one on symptomatic and one on asymptomatic clonally propagated UCB1 pistachio leaves. Although we found other Rhodococcus spp. in the same samples from which these isolates were obtained, none was $R$. fascians. If Rhodococcus is indeed the causal agent of PBTS, then cooperativity of $R$. fascians with either $R$. kroppenstedtii or $R$. corynebacterioides is an unlikely prerequisite for PBTS disease progression.

\section{PBTS symptoms do not correlate with $R$. fascians occurrence}

Several observations lead us to question whether $R$. fascians and $R$. kroppenstedtii are the causal agents of PBTS. First, PBTS symptoms on pistachio differ from those produced by pathogenic $R$. fascians on herbaceous dicotyledonous plants, which are characterized by shoot proliferation and leafy galls

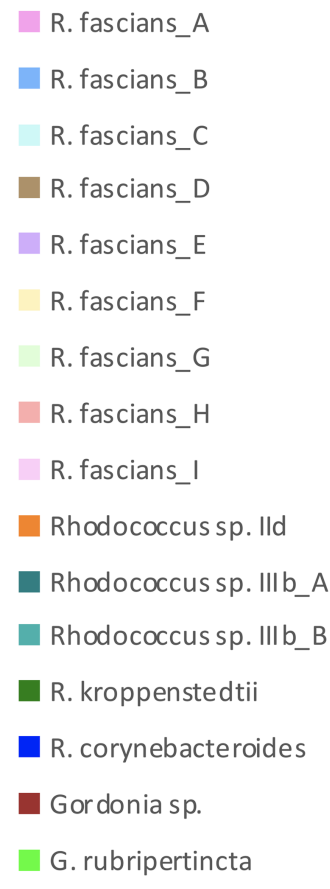

Rhodococcus_new
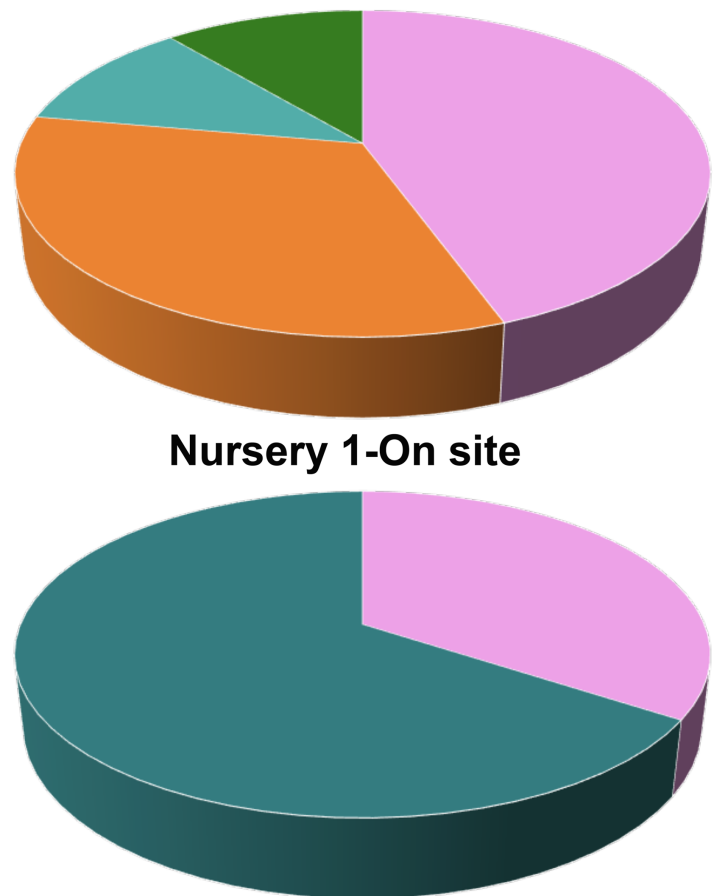

Nursery 2-On site

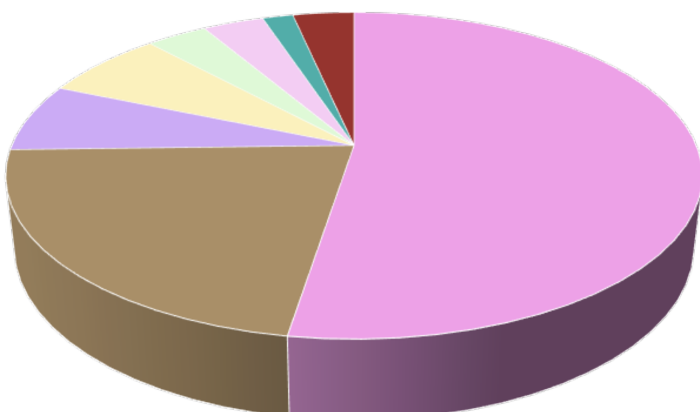

Nursery 1-Orchards

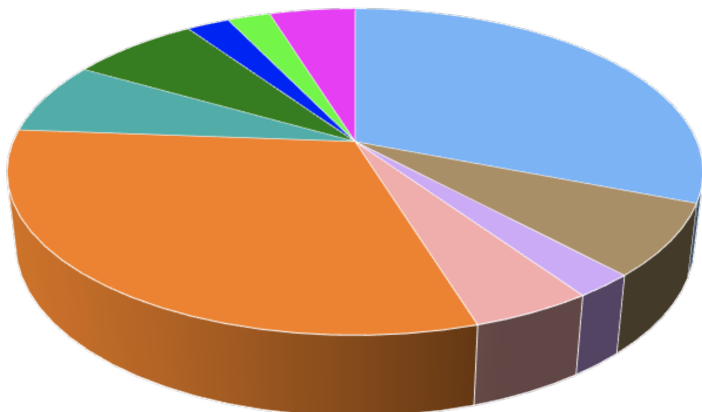

Nursery 2-Orchards

FIGURE 6

The bacterial communities differ between pistachio-producing nurseries and the orchards that they supply. Pie graphs depict the relative abundance of each Rhodococcus genotype identified from either Nursery 1 or Nursery 2 ("On site"), or from orchards that received their trees from Nursery 1 or Nursery 2 ("Orchards"). Trees from the orchards supplied by Nursery 1 contained numerous Rhodococcus genotypes that are not found in Nursery 1 . The strains isolated from trees in Nursery 2 are different from all strains found in the orchards that were supplied by Nursery 2 . The key on the left denotes the color representing the genotypes in the pie charts. $N=9$ for Nursery $1-$ On site, $n=59$ for Nursery $1-$ Orchards, $n=38$ for Nursery $2-$ On site, and $n=42$ for Nursery $2-$ Orchards. 
(Putnam and Miller 2007). Based on our observations from two disease outbreaks, and in agreement with Francis and Vereecke (2019), the main symptoms observed in affected orchards include eccentric branching, cracking of the bark, and the occurrence of gall-like protuberances on the trunk, generally developing at the graft union and at the junction between lateral branches and the main stem. In addition, most trees affected with PBTS failed to accept the scion bud following multiple grafting attempts. Previous reports characterizing the responses of plants inoculated with PBTS1 and PBTS2 only characterized a subset of these symptoms (Stamler et al. 2015; Vereecke et al. 2020). Although stunting and some root abnormalities were observed in UCB-1 plantlets inoculated with high titers of PBTS1 and PBTS2, neither galls nor cracks, the most characteristic symptoms of PBTS, were reported. Instead, slower growth rates with reduced average internode lengths were observed in plants inoculated with the Rhodococcus bacteria (Stamler et al. 2015).

Furthermore, in the current study, we failed to find a correlation between the presence of Rhodococcus species or groups and the occurrence of PBTS symptoms. Indeed, similar strain diversity was obtained from both symptomatic and asymptomatic hosts, and more than half of the $R$. fascians isolates we cultured came from asymptomatic tissue. A recent survey of Tunisian nut trees also observed that $R$. fascians was a frequent colonizer of both symptomatic and asymptomatic almond and pistachio trees (Dhaouadi et al. 2021). There was a similar lack of correlation between the genotypes of Rhodococcus strains observed in orchards and the genotypes of Rhodococcus obtained from the respective donor nurseries. Instead, Rhodococcus is a diverse, widely distributed, and endemic taxon that occurs epiphytically on a range of native and introduced host species.

The cosmopolitan nature of Rhodococcus is consistent with previous studies that revealed diverse Rhodococcus species as established members of the plant microbiome (Bai et al. 2015; Francis and Vereecke 2019). The plant-associated Rhodococcus sp. Leaf225 (Fig. 1) was isolated from leaves of wild Arabidopsis thaliana in Switzerland (Bai et al. 2015). R. corynebacterioides has been reported as a common inhabitant of the apple phyllosphere (He et al. 2012). Rhodococcus was shown also to be part of the endophytic bacterial community on grapevines (Lopez-Fernandez et al. 2017) and retrieved from the phyllosphere of poplars (Crombie et al. 2018). Additional studies have indicated the occurrence of Rhodococcus bacteria in the humus layer of Norway spruce stands (Elo et al. 2000). The current work builds upon these studies and reveals that Rhodococcus is a common epiphyte on diverse tree species in and around nurseries and commercial orchards. We observed multiple Rhodococcus species in a riparian tree stand containing several different tree

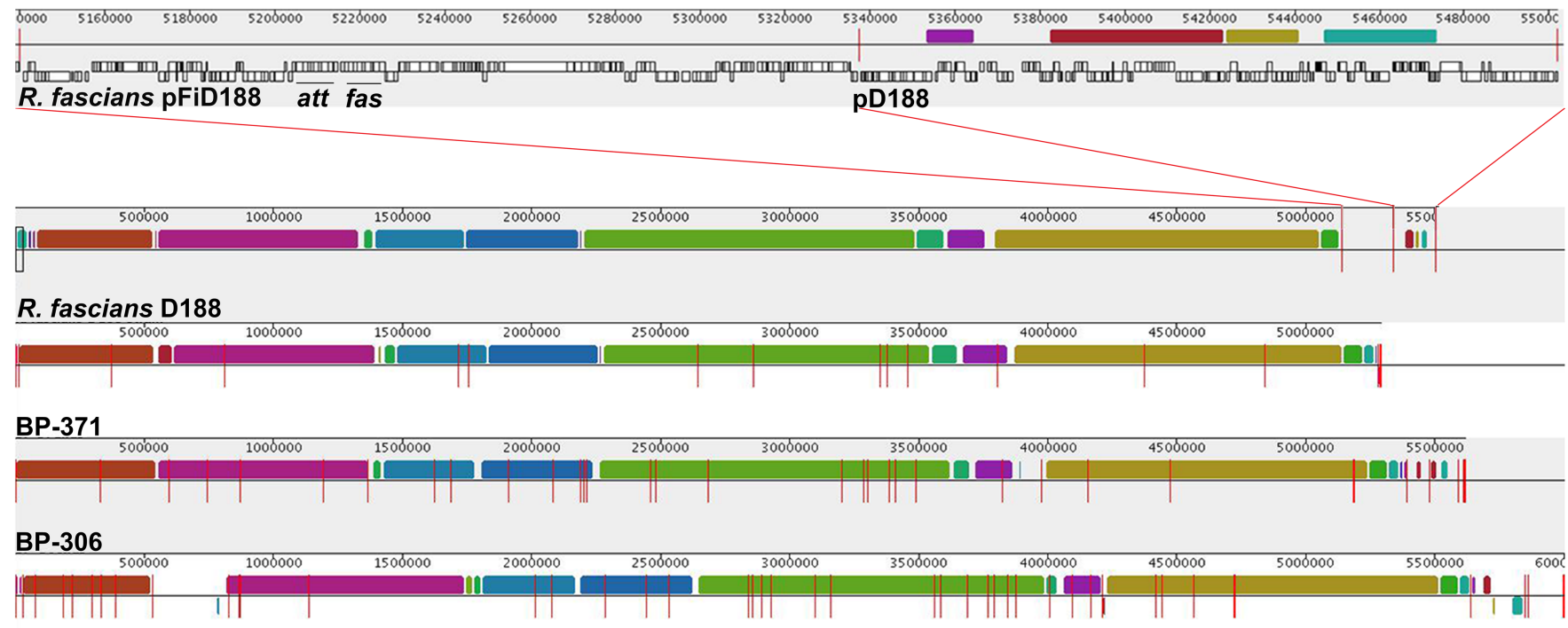

\section{BP-211}

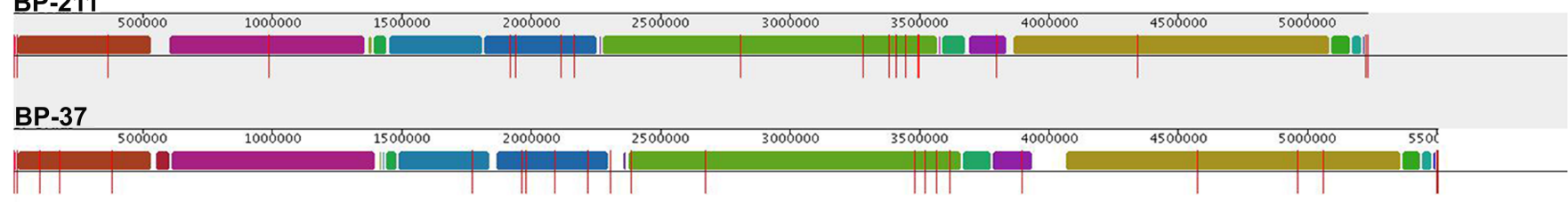

BP-197

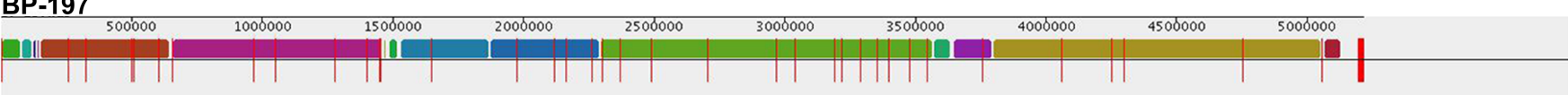

BP-181

FIGURE 7

Representative Rhodococcus fascians isolates do not contain the linear virulence plasmid pFiD188. Draft assemblies of the indicated genomes were reordered and aligned with the D188 reference genome using MAUVE. Homologous locally colinear blocks (LCBs) have the same color, with gaps between LCBs representing regions without homology. Vertical red lines in the D188 assembly demarcate boundaries between the chromosome pFiD188 and pD188. Vertical red bars in the draft genome assemblies indicate boundaries between scaffolds. The top panel is an enlarged region of the D188 alignment highlighting the two plasmids. Note there are no LCBs present in the linear virulence plasmid pFiD188, indicating a lack of homologous sequences in the $R$. fascians isolates. BP-371 is from $R$. fascians Group A, BP-306 from Group B, BP-211 from Group C, BP-37 from Group D, BP-197 from Group E, and BP-181 from R. fascians Group F. 
species, none of which exhibited symptoms of PBTS or leafy gall disease. Furthermore, $R$. fascians was frequently isolated from different, asymptomatic woody fruit species in orchards that predate the PBTS outbreaks. Thus, Rhodococcus is a common component of microbial communities on diverse plant species and neither predicts nor reflects the occurrence of PBTS.

\section{Neither the linear virulence plasmid nor fasciation-associated virulence genes were observed in California pistachio orchards}

$R$. fascians can behave as a plant pathogen, an epiphyte, or a commensal (Creason et al. 2014b; Savory et al. 2017; Stes et al. 2011). The nature of this host-microbe interaction is dictated by the presence or absence of a conjugative linear plasmid, pFiD188, that includes the virulence-associated att and fas loci. Genes at the fas locus code for cytokinin biosynthesis, considered a key factor in tissue overgrowth characteristic of PBTS (Jameson et al. 2019; Pertry et al. 2009, 2010; Radhika et al. 2015). In prior work, fas genes could not be detected in the assembled draft genome sequences of either of the PBTS strains (Stamler et al. 2016), nor of four additional Rhodococcus isolates from pistachio (Savory et al. 2017). Stamler et al. (2016) suggested the linear virulence plasmid was unstable in the absence of host selection, consistent with the report by Vereecke et al. (2020) that PBTS1 and PBTS2 cultures lose the fas gene during serial passage on rich media. Interestingly, Nikolaeva et al. (2009) concluded that the severity of fasciation symptoms in peas correlated with the number of $R$. fascians cells maintaining the plasmid. By contrast, Savory et al. (2017) detected the linear virulence plasmid in 64 of 66 genome assemblies from diverse Rhodococcus species, documenting high prevalence of the plasmid. Likewise, Dhaouadi et al. (2021) sequenced three Rhodococcus genomes from both symptomatic and asymptomatic pistachio and almond trees and identified CDSs homologous to the att and fas loci in all three genomes. These studies suggest that if present, the linear virulence plasmid is sufficiently stable such that both the virulence genes and the plasmid itself should be readily identified in genome assemblies.

Here, we analyzed 231 genomes from a diverse collection of environmental Rhodococcus, including 150 R. fascians isolates derived from both symptomatic and asymptomatic pistachio trees. We used whole genome alignments, Illumina read mapping, and BLAST searches to search for virulence genes and the virulence plasmid in our genome assemblies. In no case were virulence genes detected in the assembled genomes, nor were sequences homologous to the virulence plasmid identified in the individual Illumina reads. We conclude that endemic Rhodococcus communities of California pistachio orchards lack fas loci. Moreover, based on these findings, we suggest that these virulence determinants are part of a Rhodococcus accessory genome that is absent from the collection described here.

Using an endpoint PCR assay, Vereecke et al. (2020) detected the presence of fas genes in both epiphytic and endophytic compartments. If true, it is conceivable that the lack of virulence plasmid in the current collection reflects methodological bias. A majority of our isolates were obtained using leaf prints, which preferentially sample epiphytic communities. We attempted to isolate Rhodococcus from inside plant tissues by surface sterilizing and homogenizing tissue prior to plating; however, colonies with Rhodococcus-like morphology were never recovered from surface-sterilized leaf homogenates. Sampling the surfaces of diseased bark yielded diverse Rhodococcus species, including $R$. fascians. Similar to leaf tissue homogenates, we were unable to recover Rhodococcus from homogenates of surface-sterilized bark tissue. We did, however, isolate bacterial colonies that lacked pigmentation characteristic of Rhodococcus. Thus, our protocol was sufficient to isolate epiphytic bacteria, suggesting that Rhodococcus strains associated with pistachio are primarily epiphytic.

\section{Bacterial genotypes in orchards do not always reflect those found in source nurseries}

Understanding the relationships among diverse Rhodococcus populations could provide insight into what role, if any, Rhodococcus plays in causing PBTS. In the context of the recent PBTS outbreaks, this requires understanding the relationship between the bacterial communities in nurseries suspected to be the source of Outbreaks 1 and 2, the orchards to which they provided trees, and naturally occurring communities residing outside either of these two locations. Although $R$. fascians Group A was isolated from Nursery 1 and from orchards derived from Nursery 1 rootstocks, $R$. fascians Group A was ubiquitous at sites unrelated to Nursery 1 , including on mature pistachio trees and native flora. Given this observation, a more parsimonious explanation is that an endemic, broadly distributed, and avirulent population of $R$. fascians exists in California. In fact, we encountered additional, less common $R$. fascians genotypes in orchards supplied by Nursery 1 that were not observed on site. This suggests either that these isolates were local Rhodococcus strains or, alternatively, that the populations of $R$. fascians changed at Nursery 1 since the time that Outbreak 1 trees were distributed (2011 to 2014) and our sampling on site at Nursery 1 in 2015 .

There was a much clearer separation between strains identified at Nursery 2 and those sampled from Nursery 2-supplied orchards. Isolates from Nursery 2 were predominantly $R$. fascians Group A and Rhodococcus sp. Clade IIIb Group A, whereas neither of these genotypes was found in the orchards that acquired their rootstock from Nursery 2. Instead, symptomatic trees from Nursery 2-derived orchards yielded diverse Rhodococcus genotypes not found at Nursery 2 , including several $R$. fascians genotypes identified in the Outbreak 1-associated orchards. Furthermore, symptomatic UCB1 trees sampled at Nursery 2 produced Rhodococcus strains that were neither $R$. fascians nor R. kroppenstedtii. Taken together, these data are inconsistent with Nursery 2 serving as the source of the Rhodococcus isolates collected from PBTS-afflicted trees during Outbreak 2.

In conclusion, significant controversy surrounds the recent reports that $R$. fascians and $R$. kroppenstedtii cause PBTS (Chang et al. 2018; Randall et al. 2018; Vereecke 2018). We failed to find a correlation between strains in nurseries and the orchards they supplied. Moreover, none of the strains that we isolated contained the virulence plasmid that is commonly assumed to be required for disease. Instead, we observed a diversity of Rhodococcus strains in both managed and nonmanaged situations, including symptomatic and asymptomatic trees. These observations are consistent with an endemic, broadly distributed, and avirulent $R$. fascians population in California pistachio orchards, nurseries, and nearby wild areas. Importantly, the current data are inconsistent with the notion of a point source of virulent $R$. fascians in PBTS disease.

\section{ACKNOWLEDGMENTS}

We thank Kaitlyn Kelly and Sampson Li for technical support and the California Pistachio Research Board for financial support. 


\section{LITERATURE CITED}

Argimon, S., Abudahab, K., Goater, R. J. E., Fedosejev, A., Bhai, J., Glasner, C., Feil, E. J., Holden, M. T. G., Yeats, C. A., Grundmann, H., Spratt, B. G., and Aanensen, D. M. 2016. Microreact: Visualizing and sharing data for genomic epidemiology and phylogeography. Microb. Genom. 2:e000093.

Bai, Y., Muller, D. B., Srinivas, G., Garrido-Oter, R., Potthoff, E., Rott, M., Dombrowski, N., Munch, P. C., Spaepen, S., Remus-Emsermann, M., Huttel, B., McHardy, A. C., Vorholt, J. A., and Schulze-Lefert, P. 2015. Functional overlap of the Arabidopsis leaf and root microbiota. Nature 528:364-369.

Bankevich, A., Nurk, S., Antipov, D., Gurevich, A. A., Dvorkin, M., Kulikov, A. S., Lesin, V. M., Nikolenko, S. I., Pham, S., Prjibelski, A. D., Pyshkin, A. V., Sirotkin, A. V., Vyahhi, N., Tesler, G., Alekseyev, M. A., and Pevzner, P. A. 2012. SPAdes: A new genome assembly algorithm and its applications to single-cell sequencing. J. Comput. Biol. 19:455-477.

Bolger, A. M., Lohse, M., and Usadel, B. 2014. Trimmomatic: A flexible trimmer for Illumina sequence data. Bioinformatics 30:2114-2120.

Chang, J. H., Putnam, M. L., Grunwald, N. J., Savory, E. A., Fuller, S. L., and Weisberg, A. J. 2018. Response to comments on "Evolutionary transitions between beneficial and phytopathogenic Rhodococcus challenge disease management." Elife 7.

Creason, A. L., Davis, E. W., 2nd, Putnam, M. L., Vandeputte, O. M., and Chang, J. H. 2014a. Use of whole genome sequences to develop a molecular phylogenetic framework for Rhodococcus fascians and the Rhodococcus genus. Front. Plant Sci. 5:406.

Creason, A. L., Vandeputte, O. M., Savory, E. A., Davis, E. W., 2nd, Putnam, M. L., Hu, E., Swader-Hines, D., Mol, A., Baucher, M., Prinsen, E., Zdanowska, M., Givan, S. A., El Jaziri, M., Loper, J. E., Mahmud, T., and Chang, J. H. 2014b. Analysis of genome sequences from plant pathogenic Rhodococcus reveals genetic novelties in virulence loci. PLoS One 9:e101996

Crespi, M., Messens, E., Caplan, A. B., van Montagu, M., and Desomer, J. 1992. Fasciation induction by the phytopathogen Rhodococcus fascians depends upon a linear plasmid encoding a cytokinin synthase gene. EMBO J. 11:795-804.

Crombie, A. T., Larke-Mejia, N. L., Emery, H., Dawson, R., Pratscher, J., Murphy, G. P., McGenity, T. J., and Murrell, J. C. 2018. Poplar phyllosphere harbors disparate isoprene-degrading bacteria. Proc. Natl. Acad. Sci. U.S.A. 115:13081-13086.

Darling, A. C., Mau, B., Blattner, F. R., and Perna, N. T. 2004. Mauve: Multiple alignment of conserved genomic sequence with rearrangements. Genome Res. 14:1394-1403.

Dhaouadi, S., Hamdane, A. M., and Rhouma, A. 2021. Isolation and characterization of Rhodococcus spp. from pistachio and almond rootstocks and trees in Tunisia. Agronomy 11:355.

Drummond, A. J., Suchard, M. A., Xie, D., and Rambaut, A. 2012. Bayesian phylogenetics with BEAUti and the BEAST 1.7. Mol. Biol. Evol. 29: 1969-1973.

Elo, S., Maunuksela, L., Salkinoja-Salonen, M., Smolander, A., and Haahtela, K. 2000. Humus bacteria of Norway spruce stands: plant growth promoting properties and birch, red fescue and alder colonizing capacity. FEMS Microbiol. Ecol. 31:143-152

Francis, I., De Keyser, A., De Backer, P., Simon-Mateo, C., Kalkus, J., Pertry, I., Ardiles-Diaz, W., De Rycke, R., Vandeputte, O. M., El Jaziri, M., Holsters, M., and Vereecke, D. 2012. pFiD188, the linear virulence plasmid of Rhodococcus fascians D188. Mol. Plant Microbe Interact. 25: 637-647.

Francis, I. M., and Vereecke, D. 2019. Plant-associated Rhodococcus species, for better and for worse. Pages 359-377 in: Biology of Rhodococcus. H. M. Alvarez, ed. Springer International Publishing, Cham, Switzerland.

He, Y. H., Isono, S., Shibuya, M., Tsuji, M., Adkar Purushothama, C. R., Tanaka, K., and Sano, T. 2012. Oligo-DNA custom macroarray for monitoring major pathogenic and non-pathogenic fungi and bacteria in the phyllosphere of apple trees. PLoS One 7:e34249.

Hong, C. E., Jo, S. H., Moon, J. Y., Lee, J. S., Kwon, S. Y., and Park, J. M. 2015. Isolation of novel leaf-inhabiting endophytic bacteria in Arabidopsis thaliana and their antagonistic effects on phytophathogens. Plant Biotechnol. Rep. 9:451-458.

Jameson, P. E., Dhandapani, P., Song, J., Zatloukal, M., Strnad, M., RemusEmsermann, M. N. P., Schlechter, R. O., and Novak, O. 2019. The cytokinin complex associated with Rhodococcus fascians: Which compounds are critical for virulence? Front. Plant Sci. 10:674.

Kado, C. I., and Heskett, M. G. 1970. Selective media for isolation of Agrobacterium, Corynebacterium, Erwinia, Pseudomonas, and Xanthomonas. Phytopathology 60:969-976.

Li, B., Furihata, K., Ding, L. X., and Yokota, A. 2007. Rhodococcus kyotonensis sp. nov., a novel actinomycete isolated from soil. Int. J. Syst. Evol. Microbiol. 57:1956-1959.
Li, H., and Durbin, R. 2009. Making the leap: Maq to BWA. Mass Genom. 25:1754-1760.

Li, H., Handsaker, B., Wysoker, A., Fennell, T., Ruan, J., Homer, N., Marth, G., Abecasis, G., Durbin, R., and Genome Project Data Processing, S. 2009. The sequence alignment/map format and SAMtools. Bioinformatics 25:2078-2079.

Lopez-Fernandez, S., Mazzoni, V., Pedrazzoli, F., Pertot, I., and Campisano, A. 2017. A phloem-feeding insect transfers bacterial endophytic communities between grapevine plants. Front. Microbiol. 8:834

Mayilraj, S., Krishnamurthi, S., Saha, P., and Saini, H. S. 2006. Rhodococcus kroppenstedtii sp. nov., a novel actinobacterium isolated from a cold desert of the Himalayas, India. Int. J. Syst. Evol. Microbiol. 56:979-982.

Nikolaeva, E. V., Kang, S., Olson, T. N., and Kim, S. H. 2012. Real-time PCR detection of Rhodococcus fascians and discovery of new plants associated with $R$. fascians in Pennsylvania. Plant Health Progress 13. doi: 10.1094/PHP-2012-0227-02-RS.

Nikolaeva, E. V., Park, S. Y., Kang, S., Olson, T. N., and Kim, S. H. 2009. Ratios of cells with and without virulence genes in Rhodococcus fascians populations correlate with degrees of symptom development. Plant Dis. 93:499-506.

Page, A. J., Cummins, C. A., Hunt, M., Wong, V. K., Reuter, S., Holden, M. T., Fookes, M., Falush, D., Keane, J. A., and Parkhill, J. 2015. Roary: Rapid large-scale prokaryote pan genome analysis. Bioinformatics 31 : 3691-3693.

Pertry, I., Vaclavikova, K., Depuydt, S., Galuszka, P., Spichal, L., Temmerman, W., Stes, E., Schmulling, T., Kakimoto, T., Van Montagu, M. C., Strnad, M., Holsters, M., Tarkowski, P., and Vereecke, D. 2009. Identification of Rhodococcus fascians cytokinins and their modus operandi to reshape the plant. Proc. Natl. Acad. Sci. U.S.A. 106:929-934.

Pertry, I., Vaclavikova, K., Gemrotova, M., Spichal, L., Galuszka, P., Depuydt, S., Temmerman, W., Stes, E., De Keyser, A., Riefler, M., Biondi, S., Novak, O., Schmulling, T., Strnad, M., Tarkowski, P., Holsters, M., and Vereecke, D. 2010. Rhodococcus fascians impacts plant development through the dynamic fas-mediated production of a cytokinin mix. Mol. Plant Microbe Interact. 23:1164-1174

Pritchard, L., Glover, R. H., Humphris, S., Elphinstone, J. G., and Toth, I. K. 2016. Genomics and taxonomy in diagnostics for food security: Softrotting enterobacterial plant pathogens. Anal. Methods 8:12-24.

Putnam, M. L., and Miller, M. L. 2007. Rhodococcus fascians in herbaceous perennials. Plant Dis. 91:1064-1076.

Radhika, V., Ueda, N., Tsuboi, Y., Kojima, M., Kikuchi, J., Kudo, T., and Sakakibara, H. 2015. Methylated cytokinins from the phytopathogen Rhodococcus fascians mimic plant hormone activity. Plant Physiol. 169:1118-1126.

Randall, J. J., Stamler, R. A., Kallsen, C. E., Fichtner, E. J., Heerema, R. J., Cooke, P., and Francis, I. 2018. Comment on "Evolutionary transitions between beneficial and phytopathogenic Rhodococcus challenge disease management." Elife 7

Richter, M., and Rossello-Mora, R. 2009. Shifting the genomic gold standard for the prokaryotic species definition. Proc. Natl. Acad. Sci. U.S.A. 106:19126-19131

Sangal, V., Goodfellow, M., Jones, A. L., Seviour, R. J., and Sutcliffe, I. C. 2019. Refined systematics of the genus Rhodococcus based on whole genome analyses. Pages 1-21 in: Biology of Rhodococcus. H. M. Alvarez, ed. Springer International Publishing, Cham, Switzerland.

Savory, E. A., Fuller, S. L., Weisberg, A. J., Thomas, W. J., Gordon, M. I., Stevens, D. M., Creason, A. L., Belcher, M. S., Serdani, M., Wiseman, M. S., Grunwald, N. J., Putnam, M. L., and Chang, J. H. 2017. Evolutionary transitions between beneficial and phytopathogenic Rhodococcus challenge disease management. Elife 6

Savory, E. A., Welsberg, A. J., Stevens, D. M, Creason, A. L., Fuller, S. L., Pearce, E. M., and Chang, J. H. 2020. Phytopathogenic Rhodococcus have diverse plasmids with few conserved virulence functions. Front. Microbiol. 11:1022

Seemann, T. 2014. Prokka: Rapid prokaryotic genome annotation. Bioinformatics 30:2068-2069.

Segata, N., Bornigen, D., Morgan, X. C., and Huttenhower, C. 2013. PhyloPhlAn is a new method for improved phylogenetic and taxonomic placement of microbes. Nat. Commun. 4:2304.

Serdani, M., Curtis, M., Miller, M. L., Kraus, J., and Putnam, M. L. 2013. Loop-mediated isothermal amplification and polymerase chain reaction methods for specific and rapid detection of Rhodococcus fascians. Plant Dis. 97:517-529.

Stamler, R. A., Kilcrease, J., Kallsen, C., Fichtner, E. J., Cooke, P., Heerema, R. J., and Randall, J. J. 2015. First report of Rhodococcus isolates causing pistachio bushy top syndrome on 'UCB-1' rootstock in California and Arizona. Plant Dis. 99:1468-1476.

Stamler, R. A., Vereecke, D., Zhang, Y., Schilkey, F., Devitt, N., and Randall, J. J. 2016. Complete genome and plasmid sequences for Rhodococcus 
fascians D188 and draft sequences for Rhodococcus isolates PBTS 1 and PBTS 2. Genome Announc. 4.

Stes, E., Francis, I., Pertry, I., Dolzblasz, A., Depuydt, S., and Vereecke, D. 2013. The leafy gall syndrome induced by Rhodococcus fascians. FEMS Microbiol. Lett. 342:187-194.

Stes, E., Vandeputte, O. M., El Jaziri, M., Holsters, M., and Vereecke, D. 2011. A successful bacterial coup d'etat: How Rhodococcus fascians redirects plant development. Annu. Rev. Phytopathol. 49: 69-86.

Van der Auwera, G. A., and O'Connor, B. D. 2020. Genomics in the Cloud: Using Docker, GATK, and WDL in Terra, 1st Ed. O'Reilly Media, Beijing, China.
Vereecke, D. 2018. Comment on "Evolutionary transitions between beneficial and phytopathogenic Rhodococcus challenge disease management." Elife 7.

Vereecke, D., Zhang, Y., Francis, I. M., Lambert, P. Q., Venneman, J., Stamler, R. A., Kilcrease, J., and Randall, J. J. 2020. Functional genomics insights into the pathogenicity, habitat fitness, and mechanisms modifying plant development of Rhodococcus sp. PBTS1 and PBTS2. Front. Microbiol. 11.

Waterhouse, R. M., Seppey, M., Simão, F. A., Manni, M., Ioannidis, P., Klioutchnikov, G., Kriventseva, E. V., and Zdobnov, E. M. 2018. BUSCO applications from quality assessments to gene prediction and phylogenomics. Mol. Biol. Evol. 35:543-548. 